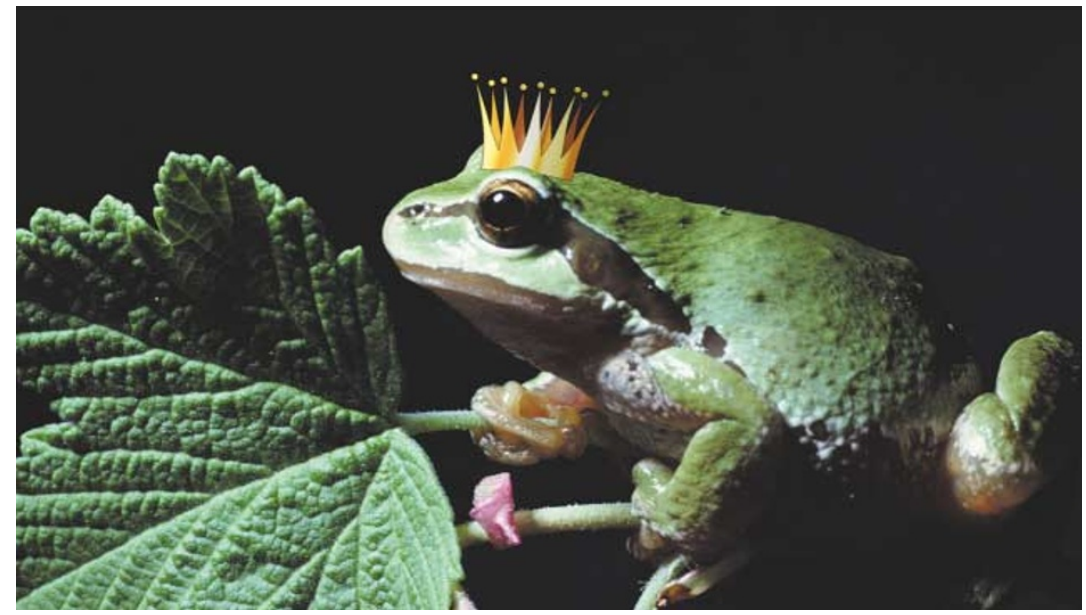

DEVELOPMENT

\section{Crowning glory}

How close are we to identifying all of the signalling pathways that are involved in neural tube patterning? The fact that the same factors keep cropping up again and again might indicate that we are getting near. For example, the signals that specify dorsal neural fates were relatively unexplored until recently, but the pathways uncovered so far consist of familiar molecules such as the Wnts and bone morphogenetic proteins (BMPs). However, as reported in Neuron, the discovery of a new signalling molecule by Tsuda et al. indicates that there might still be a few surprises in store.

The authors set out to find genes that encode secreted molecules that are involved in the patterning of the anterior central nervous system. They identified one such gene that was expressed in a crescent, or 'tiara', in the anterior ectoderm of the gastrulastage embryo, and they named this gene Tiarin. Later in development, Tiarin was expressed in the nonneural ectoderm that flanks the prospective brain region, and transcripts were also detected in the roof plate after neural tube closure.

To investigate the activity of this new protein, Tsuda et al. took eightcell Xenopus embryos and injected Tiarin mRNA into the cells that were fated to become neural plate. This manipulation caused an expansion of dorsal neural markers in the resulting embryos. There was no evidence of any increase in dorsal cell proliferation or ventral cell death, indicating that Tiarin is a genuine dorsalizing factor.

The authors then tested its effects on explants of uncommitted ectodermal tissue (animal caps) from blastula-stage embryos. Tiarin alone did not alter the fate of the tissue. However, if its mRNA was co-injected with that of the neuralizing factor Chordin, the caps acquired dorsal neural characteristics, whereas Chordin alone activated more ventral neural markers. So, Tiarin is not a neuralizing factor, but it can dorsalize ectoderm that has been neuralized by Chordin.

So, what is its mechanism of action? In the animal cap assay, Tiarin could override the ventralizing effect of sonic hedgehog (Shh), so it is tempting to speculate that it acts simply as a Shh antagonist. However, it was also able to dorsalize animal caps that did not express Shh, and it had no effect on the expression of downstream genes in the Shh pathway, such as Patched and Gli1. The authors also explored the possibility that Tiarin belongs to a Wnt or BMP signalling pathway, but they found that its expression had no significant effect on the activity of either of these pathways, and it also activated a different set of marker genes.

This raises the tantalizing possibility that Tiarin is part of a completely new signalling pathway that provides dorsalizing signals specifically for the brain neuroectoderm. If this is true, the identification of the other factors that participate in this pathway should provide an exciting new line of research.

Heather Wood

\section{(9) References and links} ORIGINAL RESEARCH PAPER Tsuda, H. et al. Dorsalization of the neural tube by Xenopus Tiarin, a novel patterning factor secreted by the flanking (2002)

FURTHER READING Lee, K. J. \& Jessell, T. M. The specification of dorsal cell fates in the vertebrate central nervous system. Annu. Rev. Neurosci. 22, 261-294 (1999) nonneural head ectoderm. Neuron $\mathbf{3 3}, 515-528$

\section{G-PROTEIN-COUPLED RECEPTORS}

\section{Orphan's pain}

'Orphan' G-protein-coupled receptors (GPCRs) — receptors that have been identified by genomic techniques, but whose function and ligands are unknown - are proving to be fertile ground for researchers who are looking for potential new drug targets. Writing in Nature Neuroscience, Lembo et al. describe how they have reunited one group of orphan GPCRs with their ligands, and uncovered a new receptor system that might be important as a potential target for analgesia.

The family of GPCRs that are described by Lembo and colleagues is expressed only in small sensory neurons in the dorsal root ganglia and trigeminal ganglia in rats and humans, which led the researchers to name these GPCRs the sensory-neuron-specific receptors (SNSRs). The neurons in which the SNSRs are expressed are involved in the transmission of pain signals. When the researchers searched for ligands for the SNSRs by expressing them in cultured cells and testing a panel of candidates, they discovered that the receptors were activated by a range of opioid-related peptides.

The most potent ligand was bovine adrenal-medulla peptide 22 (BAM22), which is produced by cleavage of the opioid peptide precursor proenkephalin A. BAM22 is expressed in the rat dorsal root ganglion and the dorsal horn of the spinal cord. It has been implicated in nociception, and can bind to all the known opioid receptors, but its function is unclear.

When Lembo et al. compared the abilities of different opioidrelated peptides to activate the SNSRs, they found that the structural requirements for activation of SNSRs were different from the requirements of opioid receptors. They also found that, unlike opioid receptors, the SNSRs could not be blocked by naloxone or other opioid-receptor antagonists. They propose that the SNSRs represent a new family of GPCRs - distinct from the opioid receptors - that are likely to be involved in nociception.

Recently, Dong et al. described a family of mouse GPCRs, called MRGs (mas-related genes), and Lembo et al. report that these are identical to the SNSRs. However, Dong et al. found that the neural MRGs, which were also expressed in a specific subset of sensory neurons, were most potently activated by peptides such as neuropeptide FF (NPFF), which has also been implicated in pain systems, but which activates the SNSRs only weakly. As there are many MRGs, it is possible that their sensitivities to NPFF and BAM22 vary.

The discovery of a family of receptors that is expressed only in nociceptive neurons, and which recognizes an opioid-type ligand through a non-opioid mechanism, might provide a new target for analgesic therapies. In particular, as the authors point out, the very specific localization of the SNSRs to the nociceptive neurons might indicate that drugs that target these receptors will have fewer side effects than compounds that target opioid receptors, which are widely expressed in the brain.

Rachel Jones

(9) References and links

ORIGINAL RESEARCH PAPER Lembo, P. M. C. et al. Proenkephalin A gene products

activate a new family of sensory neuron-specific GPCRs. Nature Neurosci. 5, 201-209 (2002) FURTHER READING Dong, $X$. et al. A diverse family of GPCRs expressed in specific subsets of nociceptive sensory neurons. Cell 106, 619-632 (2001)

WEB SITES

Encyclopedia of Life Sciences: http://www.els.net/

G protein-coupled receptors | opiate receptors 